\title{
MVDC Network Balancing for Increased Penetration of Low Carbon Technologies
}

\author{
Lewis Hunter \\ Department of Electronic and Electrical Engineering \\ University of Strathclyde \\ Glasgow, Scotland \\ lewis.hunter.100@strath.ac.uk \\ Stephen Finney \\ Department of Electronic and Electrical Engineering \\ University of Edinburgh \\ Edinburgh, Scotland \\ stephen.finney@ed.ac.uk
}

\author{
Campbell Booth \\ Department of Electronic and Electrical Engineering \\ University of Strathclyde \\ Glasgow, Scotland \\ campbell.d.booth@strath.ac.uk \\ Adrià Junyent Ferré \\ Department of Electronic and Electrical Engineering \\ Imperial College \\ London, UK \\ adria.junyent-ferre@imperial.ac.uk
}

\begin{abstract}
This paper outlines the case for using Medium Voltage Direct Current (MVDC) (5-50 kV) elements in distribution systems as a means to accelerate the deployment of low carbon technologies. The approach uses existing cable and overhead assets, originally designed into the ac system for security purposes. By selective conversion to dc, an inter GSP (grid supply point) balancing network can be created with modifications only required at substations. This approach allows for increased network transfer capability without increasing fault level as would be the case with conventional interconnection.
\end{abstract}

Using data from a real-world Scottish suburban distribution network, the benefits and barriers to such an approach are examined. Power flows for the existing network are benchmarked under various present day and future loading scenarios. Controllable MVDC links are introduced to the network and power flow studies used to assess the value of such an approach to network reinforcement. Cost estimates for such a scheme are projected using data from industrial trials.

Index Terms-MVDC, Power distribution, Power system interconnection, Smart grids

\section{INTRODUCTION}

The electrification of transport and heat, substantial increases in distributed renewable generation and predicted uptake of grid scale battery storage all pose challenges and opportunities for distribution system operators (DSO) as they evolve from distribution network operators (DNO). Issues of note include capacity constraints, increased number of voltage excursions, lower supply security and reduced power quality.

Ambitious climate change targets set by the Scottish government [1] [2] have seen a rapid growth in renewable energy generation over the last decade, predominantly onshore wind. Recently, the Scottish Government have outlined their intention to ban the sale of all petrol and diesel only vehicles by 2032 [1]. This will inevitably have pronounced impacts on distribution networks if the uptake of low emission vehicles is as dramatic as the uptake in renewables. With a significant proportion of grid supply points (GSP) in Southern and Central Scotland already restricting the connection of further distributed resource (Figure 1), due to fault level being too high or capacity concerns [3], it is clear that new capacity urgently needs to be released into these networks.

The creation of additional conventional ac network capacity is a time consuming and expensive exercise due to planning timescales, inflating commodity costs, long fabrication lead times and poor cost-benefit of 'more copper' reinforcement strategies. Investment maps for DNOs already show relatively small ( $£ 100 \mathrm{k}-£ 500 \mathrm{k})$ modernisation and uprating projects being actively worked on which are not due for completion until 2023 [4].

With the expected rapid uptake of low carbon technologies, including the transition to EVs, a quicker means of performing capacity upgrades is clearly required [5].

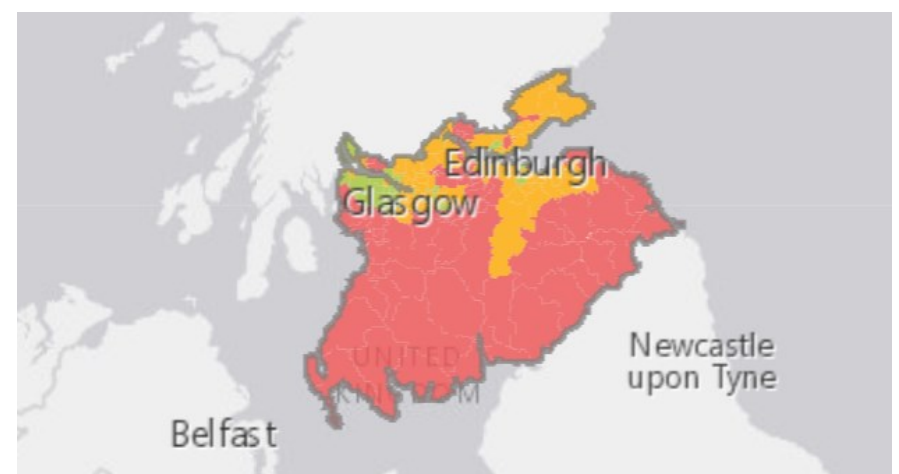

Figure $1-33$ and $11 \mathrm{kV}$ generation heat map of Central and Southern Scotland. red areas outline regions where at least one factor is close to its operational limit, amber areas illustrate areas where at least one factor is approching its operation limit and green regions have no constraining factors (within reason) [3] 
Power electronics are no longer the expensive option they once were [6] relative to cables, lines and transformers. This changes the way that network reinforcements can be carried out. The role of high voltage dc (HVDC) and low voltage dc (LVDC) has already increased significantly over the past two decades and are well explored both industrially and academically [7]. Controllability has led to the majority of domestic appliances now inherently being dc (interfaced to grid via switch mode power supplies). The properties of HVDC have made it commonplace for the technology to be used for long distance transfers due to the greater power densities achieved and the reduction of line losses. MVDC, on the other hand, is a significantly less explored area. MVDC may be a means of unlocking latent capacity in existing distribution networks without encountering collateral, negative impacts such as increasing fault level beyond safe operational limits.

\section{STUDY OVERVIEW}

Through a series of scenario-based load flow studies, the benefits of 'network balancing' formed by the interconnection of GSPs using MVDC have been identified. Using indicative costs from demonstrator projects, an estimate for such reinforcement has been made and compared to an equivalent $33 \mathrm{kV}$ ac reinforcement. Technology readiness level and present industrial activity has been assessed. From the studies conducted it has been shown that even limited controllable interconnection makes a significant different to wider network transfers.

Modelling is carried out using DIgSILENT PowerFactory 15.2. Similar studies have looked at the role that single embedded MVDC links could play in distribution networks to create additional export capacity [8] - [10].

\section{NETWORK CASE STUdiES}

\section{A. Network Design Overview}

Figure 2 and Table I outline a simplified network topology and device ratings to help contextualise the modelling. A more detailed description of Scottish network design philosophy is available [11] [12].

Due to security requirements set out by Engineering Recommendation P2-6 [13] and 'the Distribution Code for Licensed DNOs of Great Britain' [14], high voltage networks (11 kV and upwards) are designed to an N-1 standard. This means that the network should continue to operate as normal with the loss of one asset. The use of normally open and closed points allows for network reconfiguration to occur in the event of a planned or forced outage event. Reconfiguration is generally carried out to keep the largest group of customers online, incentivised by regulator-imposed penalties on the DNO based on customer minutes lost and customer interruption.

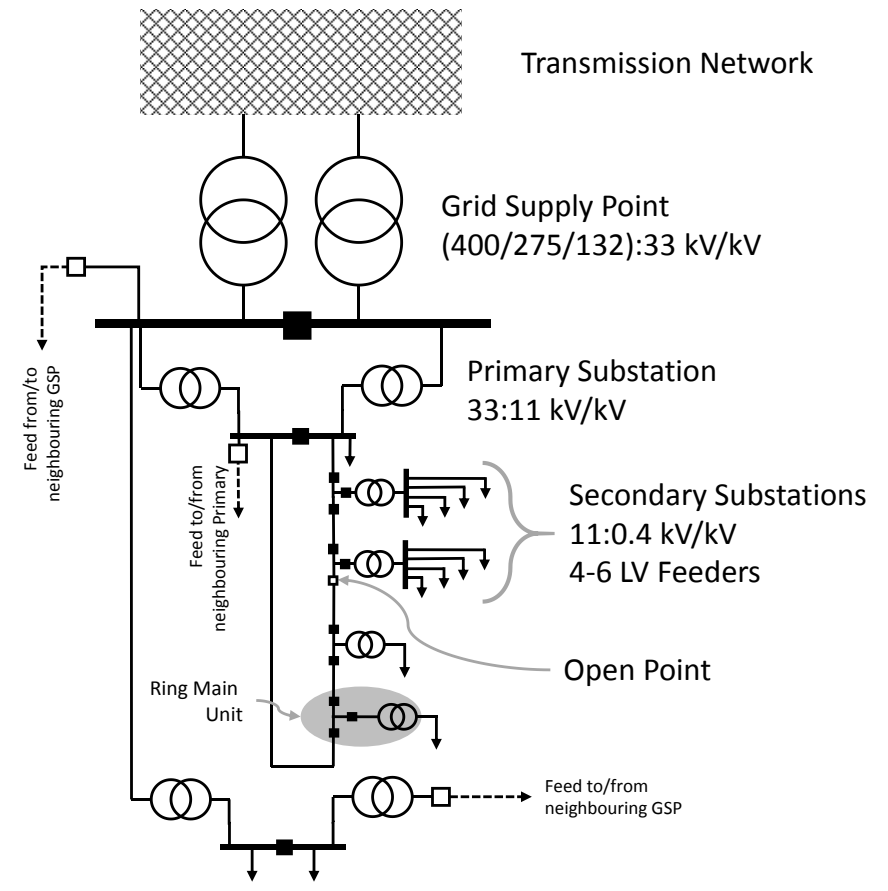

Figure 2- Typical Scottish Network Topology with substation voltages and terminology outlined

TABLE I. TYPiCAL SUBSTATION RATINGS

\begin{tabular}{|c|c|c|}
\hline Location & Voltage $(\boldsymbol{k V}: \boldsymbol{k}$ ) & Typical Rating \\
\hline Grid Supply Point & $400 / 275 / 132: 33$ & $10-240 \mathrm{MVA}$ \\
\hline Primary & $33: 11$ & $5-24 \mathrm{MVA}$ \\
\hline Secondary & $11: 0.4 / 0.23$ & $10-1000 \mathrm{kVA}$ \\
\hline
\end{tabular}

\section{B. Test Network Characteristics}

The single line schematic presented in Figure 3 shows a real world Scottish suburban distribution network. Eleven primary substations are distributed across the sector with secure ratings of between 21 and 24 MVA depending upon site. All lines have a winter maximum continuous rating of 20.86 MVA apart from 36.9 MVA for line 9. Table II outlines operating properties for GSPs. Note that the high level of distributed generation (DG) at GSP 3 means that the transformer is almost meeting its firm reverse power capability limit. A breakdown of generation for the sector can be found in Table III.

Lines 3, 4, 7, 8 and 9 all represent normally open assets which provide $\mathrm{N}-1$ security and interconnect the three grid supply points within the sector spanning distances of up to 7.9 $\mathrm{km}$ in the case of line 9. Conductors (7/8 and 3/4 form double circuit overhead routes while line $\mathbf{9}$ is a single circuit. As alluded to previously, these lines are present in the network for fault restoration and maintenance purposes. Were these assets to be closed under normal operation it is likely that the fault level would be greater than the designed limit of 1,000 MVA [15]. During normal operation these assets sit idle and financially could be considered as 'part-stranded'. These redundant, non-load carrying, assets will form the backbone to the proposed MVDC balancing network 


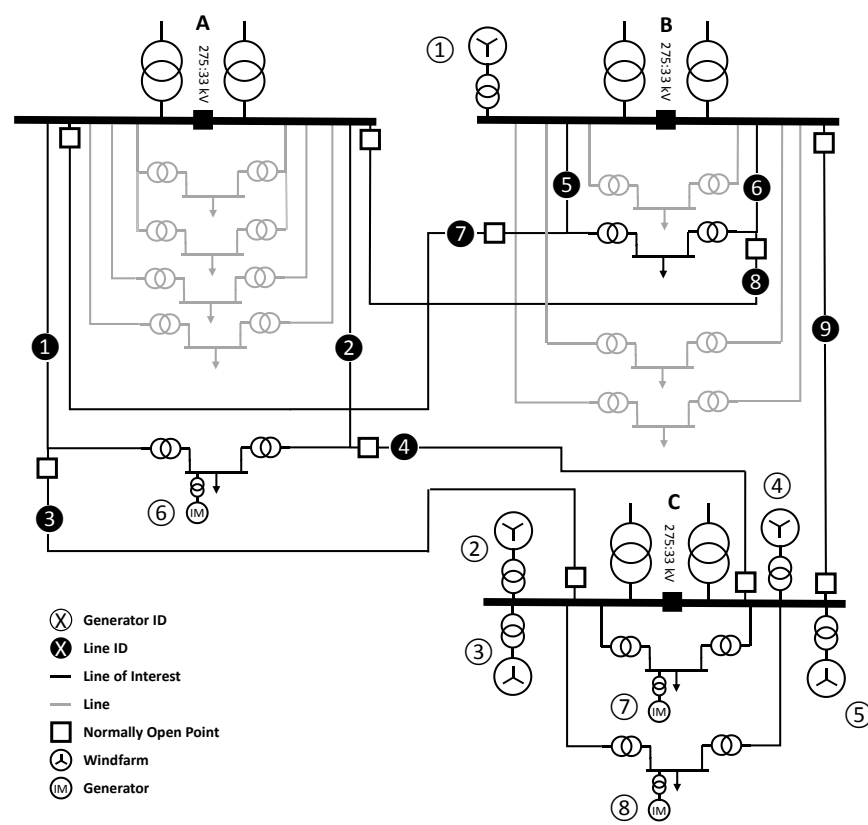

Figure 3 - Simplified distribution network sector comprising of three GSP all of which are interconnected via normally open points.

TABLE II. GSP PROPERTIES

\begin{tabular}{|c|c|c|c|}
\hline & \multicolumn{3}{|c|}{ Electrical Properties } \\
\hline ID & $\begin{array}{c}\text { Maximum } \\
\text { Continuous } \\
\text { Rating } \\
(\boldsymbol{M V A})\end{array}$ & $\begin{array}{c}\text { Connected } \\
\text { DG } \\
(\boldsymbol{M V A})\end{array}$ & $\begin{array}{c}\text { Mean } \\
\text { GSP Loading } \\
\text { (2016) } \\
(\mathbf{M V A})\end{array}$ \\
\hline $\mathrm{A}$ & $120^{\mathrm{a}}$ & 0.5 & 34.2 \\
\hline $\mathrm{B}$ & $120^{\mathrm{a}}$ & 12.3 & 23.6 \\
\hline $\mathrm{C}$ & $120^{\mathrm{a}}$ & 110.3 & $-21.8^{\mathrm{b}}$ \\
\hline \multicolumn{5}{|c|}{ b. Negative values indicate an exporting node } \\
\hline
\end{tabular}

TABLE III: DISTRIBUTED RESOURCE RATING

\begin{tabular}{|c|c|c|}
\hline $\begin{array}{c}\text { Distributed } \\
\text { Resource } \\
\text { Type and } \\
\text { Rating }\end{array}$ & \multicolumn{2}{|c|}{ Distributed Resource Characteristics } \\
\hline ID & DG Rating $($ MVA $)$ & Type \\
\hline 1 & 12.3 & Onshore Wind \\
\hline 2 & 26 & Onshore Wind \\
\hline 3 & 30 & Onshore Wind \\
\hline 4 & 11.5 & Onshore Wind \\
\hline 5 & 39 & Onshore Wind \\
\hline 6 & 0.5 & Run of River Hydro \\
\hline 7 & 2 & Waste Incineration \\
\hline 8 & 4.2 & Waste Incineration \\
\hline
\end{tabular}

For modelling purposes, the $11 \mathrm{kV}$ and $0.4 \mathrm{kV}$ distribution networks are modelled as lumped $11 \mathrm{kV}$ real and reactive power injections or demands. Conductors are modelled as lumped (R-L) element impedances. $11 \mathrm{kV}$ bus voltages are regulated to $\pm 0.03 \mathrm{pu}$ from nominal via automatic tap changers located at primary substations for a simulation to have a successful network solution.
Using load and generation data for the distribution sector, five benchmark states (a-e) listed subsequently have been modelled to understand network behaviour. Maximum generation assumes all units are at full output resulting in 125.5 MVA of generation across the sector; for minimum generation it is assumed all units have zero output. Line and GSP loading for the outlined scenarios are presented in Table IV. The $20 \%$ demand increase associated with scenario e is consistent with predictions made by the system operator, National Grid [16].
a. Maximum demand and maximum generation
b. Minimum demand and maximum generation
c. Minimum demand and minimum generation
d. Maximum demand and minimum generation.
e. Maximum demand (2040) and minimum generation.

TABLE IV. SECTOR LOADING BY SCENARIO (NEGATIVE INDICATES AN EXPORTING GSP)

\begin{tabular}{|c|c|c|c|c|c|}
\hline & \multicolumn{5}{|c|}{ Pcenario } \\
\cline { 2 - 6 } Asset & $\boldsymbol{a}$ & $\boldsymbol{b}$ & $\boldsymbol{c}$ & $\boldsymbol{d}$ & $\boldsymbol{e}$ \\
\hline $\mathrm{A}$ & 54.9 & 13.2 & 13.7 & 55.4 & 66.9 \\
\hline $\mathrm{B}$ & 35.2 & 1.0 & 10.5 & 44.8 & 55.3 \\
\hline $\mathrm{C}$ & $-83.0^{\text {a }}$ & -93.0 & 3.3 & 13.8 & 16.7 \\
\hline $\mathbf{1} / \mathbf{2}$ & 39.1 & 8.1 & 9.3 & 40.7 & 50.7 \\
\hline $\mathbf{3} / \mathbf{4}$ & & & 4.3 & 17.6 & 21.3 \\
\hline $\mathbf{5} / \mathbf{6}$ & 17.6 & 4.3 & & & \\
\hline $\mathbf{7} / \mathbf{8}$ & & & \multicolumn{5}{|c|}{ Negative values indicate an exporting node } \\
\hline $\mathbf{9}$ & & \multicolumn{5}{|c|}{} \\
\hline
\end{tabular}

While GSP A and B are lightly to moderately loaded for all scenarios, GSP $\mathrm{C}$ has a wide range of operation points from nearly full export to low levels of import depending upon the state of the embedded wind generation. GSP A and B are load serving. An increased load at GSP C would help reduce GSP C export but the physical geography of the site makes this unlikely.

\section{MVDC NETWORK STUDIES}

Using the existing interconnection, the following section examines the possibility of increasing network capacity by repurposing normally open assets to controlled MVDC links. The key benefit here is that power can potentially be flexibly balanced without increasing system wide fault level further.

\section{A. MVDC Interconnection}

Through conversions of the lines as outlined in Figure 4, three MVDC bipole links operating at $\pm 27 \mathrm{kV}$ are inserted into the model to create an inter-GSP 'balancing' network. Switching losses are assumed to be fixed at $1 \%$ of converter load. The operating dc $\left(\mathrm{V}_{\mathrm{dc}}\right)$ voltage for MVDC scheme is selected based upon the ac peak phase to ground voltage as outlined in equation (1) where $V_{L L_{R M S}}$ is the rms line to line voltage, i.e. $33 \mathrm{kV}$. 


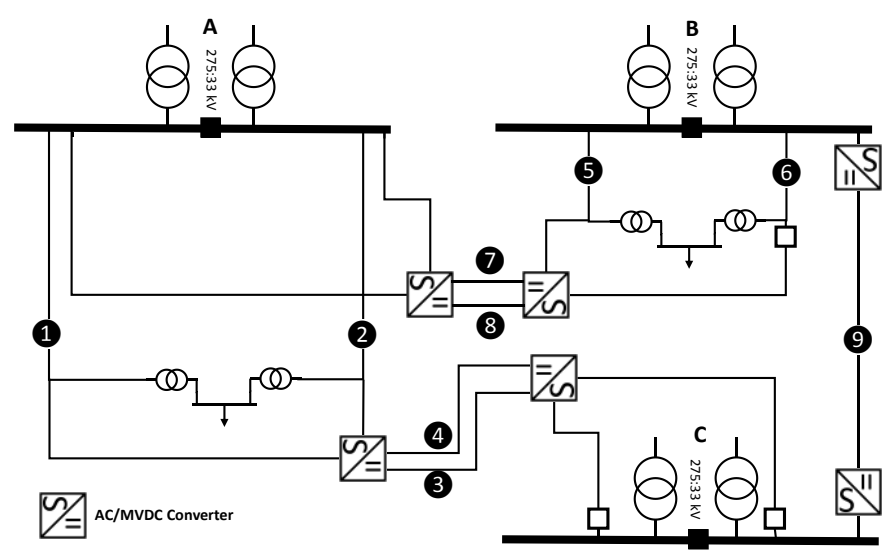

Figure 4 - Network showing MVDC interconnection between GSPs. Note that generation and primary substations were removed from the diagram for clarity purposes but remained connected for network studies

$$
V_{d c}=\frac{\sqrt{2} \times V_{L L_{R M S}}}{\sqrt{3}}
$$

For simulations, the conductor ratings of dc network elements are not increased; therefore, any benefit of introducing controlled power electronic links is purely a control advantage for these scenarios. Power converters are rated to line thermal limits. Note that because circuits 3/4 and 7/8 are double circuit overhead lines the circuit ratings are combined when modelling to form a circuit with twice the capacity of the ac winter continuous rating $(2 \times 20.86 \mathrm{MVA}=41.72 \mathrm{MVA})$.

It is acknowledged that an increase in conductor transfer capacity would be expected in the conversion of double circuit schemes, the conversion of a three wire single circuit is a tradeoff between converter topology (and therefore cost) and power transfer capacity gained.

\section{B. MVDC Studies}

The following scenarios (f-i) are executed with the three MVDC links placed on the network. Result for the studies are shown in Table V.

f. Minimum demand and max generation with GSP export to be balanced

g. Maximum demand and increased renewables generation (of $99 \mathrm{MVA}$ ) at GSP $\mathrm{C}$ such that the MVDC converters are at full export and that GSP C has reached its firm capacity.

h. Minimum demand and increased renewables generation (of $87 \mathrm{MVA}$ ) at GSP $\mathrm{C}$ such that the MVDC converters are at full export and that GSP C has reached its firm capacity.

i. A $40 \mathrm{MW}$ point load interfaced with an inverter placed midway along the dc link 3/4 fed equally from GSP $\mathrm{A}$ and $\mathrm{C}$ under minimum demand and maximum generation conditions j. Same scenario as i but with an additional $40 \mathrm{MW}$ point load situated mid-way on de link 9.

TABLE V. MVDC BALANCING NETWORK STUDIES

\begin{tabular}{|c|c|c|c|c|c|}
\hline & \multicolumn{5}{|c|}{ Scenario } \\
\cline { 2 - 6 } & \multicolumn{5}{|c|}{ Percentage Asset Loading (\%) } \\
\hline Asset & $f$ & $g$ & $h$ & $i$ & $j$ \\
\hline $\mathrm{A}$ & -25.2 & 22.3 & 10.4 & 30.2 & 30.1 \\
\hline $\mathrm{B}$ & -25.4 & 8.5 & -29.7 & 1 & 17.4 \\
\hline $\mathrm{C}$ & -27.5 & -100 & -100 & -71.8 & -55.2 \\
\hline $\mathbf{1} / \mathbf{2}$ & 89.4 & 64.1 & 89.4 & 56.9 & 56.9 \\
\hline $\mathbf{3} / \mathbf{4}$ & 100.0 & 100.0 & 100.0 & 50.0 & 50.4 \\
\hline $\mathbf{5} / \mathbf{6}$ & 16.4 & 17.6 & 4.3 & 4.3 & 4.3 \\
\hline $\mathbf{7} / \mathbf{8}$ & 21.1 & 0.0 & 0.0 & 0.0 & 0.0 \\
\hline $\mathbf{9}$ & 100.0 & 100.0 & 100.0 & 0.0 & 54.4 \\
\hline
\end{tabular}

\section{Result Discussion}

From studies $\mathrm{g}$ and $\mathrm{h}$, additional export capacity for the connection of more DG on the network is created at GSP C via the controlled interconnection with GSP A and C. While keeping within the firm capacity of GSP C, under maximum generation and minimum demand scenarios, an additional $87 \mathrm{MW}$ can connect.

Another advantage of MVDC interconnection is the ability to share dynamically (and balance) export capacity as demonstrated in study f. Studies $i$ and $j$ show how large point loads can connect to the network and can be dynamically loaded across grid supply points.

\section{MVDC ChaLLENGES AND PILOTS}

MVDC has been mooted by many sectors (e.g. mining [17], shipboard [18], offshore wind generation [19]) to reduce system losses, exploit existing assets further and to improve network control. While MVDC research and deployment for shipboard applications is widespread there is significantly less attention looking at using MVDC for land-based network reinforcement. However, interest has started growing in recent years with large multinational engineering companies and original equipment manufacturers (OEM) now offering solutions in this field [20] [21].

\section{A. Angle-DC}

Encouraged by recent industrial offering in the MVDC sphere, the UK energy regulator, OFGEM, awarded funding to a DNO (SP Energy Networks) via their Network Innovation Competition to develop and deliver the UK's first MVDC demonstrator project [10] (Angle-DC). The project budget of $£ 14.8$ million will see the delivery of an MVDC link which connects two adjacent network sections with the aim of increasing the export capability of the area (thus allowing a greater penetration of distributed renewable generation).

The converters will be of multi-modular converters (MMC) configuration allowing full control of real and reactive powers at both ends of the link. The scheme is to have a bipolar configuration operating at a dc voltage of $\pm 27 \mathrm{kV}$ with approximately $35 \mathrm{MVA}$ of transfer capability. The two poles of 
the dc link take geographically different routes formed by the combining of the conductors of the existing three wire ac schemes thus avoiding pole to pole faults. The circuits are predominantly cable with small overhead sections [22].

Initial estimates by the DNO suggest that a $20 \%$ reduction in network losses is to be expected ( $£ 630 \mathrm{k}$ per annum saving) upon successful energization [10]. SP Energy Networks hope to move the technology readiness level of such a solution from $5 / 6$ to $7 / 8$. The scheme is due to be energized in early 2019 .

\section{B. Other Developments}

MVDC activity has also been mooted and investigated for electrification of remote communities in Alaska and Canada where distribution level loads (1-10 MW) would need to be supplied via transmission scale infrastructure $(138 \mathrm{kV})$ due to charging currents and line losses [23]. A small scale $250 \mathrm{~kW}$ converter was trailed as part of this exercise.

A scoping exercise has been undertaken looking into the benefits, practicality and delivery consideration for a MVDC based distribution network at the RWTH Aachen University Campus, Germany [24]. The proposed system would consist of five main power conversion nodes interfacing between $\pm 5 \mathrm{kV}$ $\mathrm{dc}$ to three phase loads with ratings between 1 and $5.5 \mathrm{MW}$. One node has grid connects the campus network to the main 20 kV DNO supply. Another would house a $5 \mathrm{MW} / \mathrm{MWh}$ battery.

\section{Challenges}

While the benefits of repurposing existing assets to MVDC have been outlined there are many potential barriers to overcome both technically and regulatory. A non-exhaustive selection of five such challenges are briefly outlined.

\section{1) Efficiency}

While the correct placement of power electronics links can significantly reduce network losses, the efficiency of the power electronics converters themselves must be high. At distribution scale voltages, even a $2 \%$ loss factor is equivalent to hundreds of gigawatt hours per annum. Losses are inevitable but managing them, in particular the heat associated with the power electronic switching, is a must. This is likely to mean positive pressure, climate-controlled server style clean room areas being required in substations.

\section{2) Footprint}

With some GSPs perhaps only being $1000 \mathrm{~m}^{2}$ and a primary substation being $500 \mathrm{~m}^{2}$, fitting the required MVDC converters into existing sites poses challenges to manufacturers. While the size of power electronics themselves may not be large, the volume associated with the required cooling plant, transformers and control systems can be considerable. Designers need to be mindful of the power density of the entire system while keeping the necessary clearance between assets.

\section{3) Investments structure}

Existing grid infrastructure has been financially discounted over an operational time of many decades (30 to 40 years being commonplace). With power electronic machine drives perhaps having a manufacturer warranty of five years, there is a challenge for DNO/DSOs, should they wish to deploy MVDC, in setting a commercially and technically appropriate target payback period. Modularity in design and confidence in the futureproofing of MVDC deployments is therefore critical to take MVDC from an innovation level to business as usual.

\section{4) Costs}

According to the Angle-DC funding proposal, the uptake of embedded MVDC depends largely on the cost of power electronics, which SP Energy Networks estimate will drop by up to 55\% between now and 2040 [8]. With the cost per installed watt presently being $0.42 £ / W$ there is a clear challenge to reduce these down to $0.19 £ / W$.

If the three MVDC links were introduced to the test network, the direct translation of costs would be $£ 7.6$ million for each of the double circuit lines and $£ 7$ million for the single circuit. While it is appreciated that this is a crude calculation, a $£ 22$ million investment is clearly a significant expense for a DSO which needs to be fully justified with a cost benefit or least worst regret analysis.

\section{Other options?}

The simulations presented in this paper do not represent an optimal network solution but merely demonstrate the advantages of introducing controlled interconnection. A number of other options exist which could take advantage of 'part-stranded' assets which interconnect GSPs. The electricity regulator in the UK has funded DNO investigations in the use of fault current limiters [25], distribution quadrature boosters [26] as well as the 'soft normally open point' [27] (a back to back converter topology) in the hope that latent capacity can be released on distribution networks at least cost to the consumer.

\section{CONCLUSION}

Power flow analysis has shown that inter-GSP MVDC allows for increased penetration; for the situation studied this means $99 \mathrm{MW}$ in the case of maximum local demand and 87 under minimum demand. The use of existing 'part-stranded' assets used for redundancy purposes represents a promising solution to delivering additional network capacity using existing conductor wayleaves.

Using controlled MVDC links, the future DSO will have the ability to load a network sector more dynamically to allow significant increases in power flows whilst not exceeding the firm capacity of grid supply point transformers. While conventional interconnection may offer some benefits, the lack of controllability of such largely passive grids means that the power flows are dictated by the impedance map rather than by efficient routing. 
While MVDC still has a number of technical and commercial barriers to becoming a 'business as usual' approach to network reinforcement, this paper demonstrates the potential for unlocking latent capacity without adding 'more copper' to the network.

The interconnection of GSPs using dc may allow the connection of large scale point loads with capacity dynamically allocated across multiple connected generators and GSP interfaces as demonstrated with the connection in the case study presented here of two $40 \mathrm{MW}$ point loads across the MVDC network.

\section{ACKNOWLEDGMENTS}

This work has been supported through the Engineering and Physical Sciences Research Council (EPSRC) Centre for Doctoral Training in Future Power Networks and Smart Grids (EP/L015471/1).

\section{REFERENCES}

[1] The Scottish Government, "Scottish Energy Strategy: The future of energy in Scotland," Edinburgh, 2017.

[2] The Scottish Government, "2020 Routemap for Renewable Energy in Scotland," The Scottish Government, Edinburgh, 2011.

[3] SP Energy Networks, "SP Distribution Heat Map," SP Energy Networks, January 2018. [Online]. Available: https://goo.gl/X6JcwT.

[4] SP Energy Networks, "Investment \& Innovation," SP Energy Networks, February 2018. [Online]. Available: https://goo.gl/ufkmof.

[5] National Grid, "Future Energy Scenarios," 2017. [Online]. Available: http://fes.nationalgrid.com/. [Accessed 24 April 2016].

[6] R. W. D. Doncker, "The War of Currents," in International Conference on DC Microgrids, Nuremberg, 2017.

[7] IEEE/PED Transmission and Distribution Committee, "Existing HVDC Projects Listing," IEEE HVDC and FACTS Subcommittee, 2013. [Online]. Available: https://goo.gl/DyJCef. [Accessed 05 May 2017].

[8] L. Hunter, C. Booth, A. J. Ferre and S. Finney, "MVDC for enhanced utility scale distribution power delivery and control," in 52nd International Universities Power Engineering Conference (UPEC), Heraklion, 2017.

[9] TNEI, "MVDC Technology Study - Market Opportunities and Economic Impact," 16 February 2015. [Online]. Available: https://goo.gl/6xnGu6. [Accessed November 2016].

[10] SP Energy Networks, "Angle-DC 2015 Electricity Network Innovation Competition," 2015. [Online].
Available: https://goo.gl/k5Zswl. [Accessed March 2017].

[11] SSEN, "Long Term Development Statement," Scottish Hydro Electric Power Distribution, November 2016. [Online]. [Accessed 2017 January].

[12] SP Distribution, "Long Term Development Statement," November 2016. [Online]. [Accessed January 2017].

[13] The Engineering Council, "Engineering Recomendation P2/6 - Electricity Distribution Network Planning," London, 2006.

[14] DCODE, “The Distribution Code for licensed DNOs of Great Britain - Issue 29," February 2018.

[15] SP Energy Networks, "Calculation of System Fault Levels (ESDD-02-006),” 2008.

[16] National Grid, "Electricity Ten Year Statement 2017," 2017.

[17] C. Yuan, M. A. Haj-ahmed and M. S. Illindala, "Protection Strategies for Medium-Voltage DirectCurrent Microgrid at a Remote Area Mine Site," IEEE Transactions on Indstry Applications, vol. 51, no. 4, pp. 2846-2853, 2015.

[18] IEEE Industry Applications Society , "IEEE Recommended Practice for $1 \mathrm{kV}$ to $35 \mathrm{kV}$ MediumVoltage DC Power Systems on Ships," IEEE Std 17092010, 2010.

[19] D. J. G. J. J. Robinson, "Analysis and Design of an Offshore Wind Farm Using a MV DC Grid," IEEE Transactions on Power Delivery, vol. 25, no. 4, pp. 21642173, 2010.

[20] ABB, "Medium voltage direct current applications," ABB, Dalmine, 2017.

[21] Siemens, "MVDC Plus," 2017. [Online]. Available: siemens.com/mvdc.

[22] SP Energy Networks, "NIC Project Medium Voltage Direct Current Link Technical Specification," 2017.

[23] The Alaska Center for Energy and Power; University of Alaska Fairbanks, "Small-Scale High Voltage Direct Current," 2013.

[24] F. Mura and R. W. De Doncker, "Design Aspects of a Medium-Voltage Direct Current (MVDC) Grid for a University Campus," in 8th International Conference on Power Electronics - ECCE Asia, Korea, 2011.

[25] Northern Power Grid, “33kV Superconducting Fault Current Limters - Final Closedown Report," 2015.

[26] UK Power Networks, "Flexible Plug and Play; A guide to the first Quadrature-booster to be installed on the 33 kV Distribution Network," 2014.

[27] UK Power Networks, "Flexible Urban Networks; Low Voltage - Project Closedown Report," 2017. 